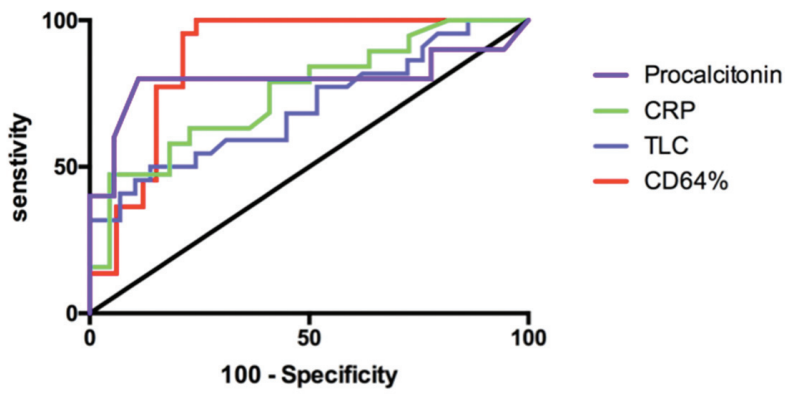

Abstract SAT0382 - Figure 1. ROC analysis of various parameters in patients with underlying SLE

Conclusions: CD64 expression on neutrophils is helpful in differentiating bacterial infection from disease flare in patients with SLE and AAV.

Disclosure of Interest: None declared

DOI: 10.1136/annrheumdis-2018-eular.7239

\section{SAT0383 RHEUMATOLOGICAL DISORDERS FOLLOWING CHIKUNGUNYA INFECTION - A RETROSPECTIVE STUDY OF 882 CASES FROM NEW DELHI}

N. Gupta. Clinical Immunology and Rheumatology, Max Super Speciality Hospital, Shalimar Bagh, New Delhi, DELHI, India

Background: Chikungunya virus (CHIKV) is notoriously arthritogenic and is known to cause a wide spectrum of rheumatological disorders.

Objectives: To study the Rheumatological Manifestations of CHIKV Infection. Methods: Record of 1641 consecutive patients who presented to the outpatient department (OPD) with history of chikungunya infection (fever, joint pains \pm rash) during the period of 1 st July 2016 to 31 st October 2016 were checked and recruited from private hospital, camps and clinic from urban and suburban population from 1 st February to 31 st December 2017. The study cohort was subjected to laboratory tests - serum IgM, IgG and RT-PCR for chikungunya virus. Those patients who tested positive for $\geq 1$ lab tests were included in the study. Of the 1641 patients, 408 patients either refused the lab tests or were lost to follow up. Of the remaining 1233 patients, $882(71.53 \%)$ patients tested positive for $\geq 1$ lab tests and $351(28.47 \%)$ tested negative for all the 3 lab tests. These patients were classified into rheumatological disorders as per validated criteria.

Results: We studied 882 consecutive patients of chikungunya infection with some rheumatological manifestations. Mean age of study cohort was 53.64 \pm 15.82 years with male:female of 398 (45.12\%):484(54.88\%). Mean disease duration of post CHIKV infection was $08.45 \pm 3.64$ months. Serologically, isolated serum IgM CHIKV was positive in 128 (14.51\%) patients, IgG CHIKV in 340 (38.54\%) patients and RT-PCR CHIKV in 223 (25.28\%) patients. Combination of serum IgG and IgM CHIKV both were positive in 62 (7.03\%), IgM and RT-PCR CHIKV 49 (5.55\%) and IgG and RT-PCR CHIKV in 40 (4.54\%) patients. All the 3 serum IgM, IgG and RT-PCR CHIKV were positive in 50 (5.67\%) patients.

Of these 882 patients, $143(16.21 \%)$ patients had rheumatological disorder prior to CHIKV infection which flared up after the infection. Of the 143 patients, 69 (48.25\%) had osteoarthritis (OA) knee, 29 (20.27\%) had mechanical low back ache, $12(8.39 \%)$ cervical spondylitis, $10(6.99 \%)$ had rheumatoid arthritis (RA), 7 $(4.89 \%)$ each adhesive capsulitis and ankylosing spondylitis (AS), 3 (2.1\%) psoriatic arthritis (PsA), 2 (1.4\%) each had SLE, dequervian tenosynovitis and granulomatosus with polyangitis.

Remaining 739 patients presented with various rheumatological disorders, most common of which was post chikungunya polyarthralgias in 568 (76.86\%) patients. Of the 739 patients, $40(5.4 \%)$ patients developed new onset OA knee, 38 (5.14\%) met ACR/EULAR 2010 criteria for RA, 28 (3.78\%) met modified New York criteria for AS, 29 (3.92\%) developed undifferentiated arthritis, 17 (2.3\%) patients developed various enthesitis and tenosynovitis, $1(0.14 \%)$ patients fulfilled ACR 1997/SLICC 2012 criteria for SLE, 20 (2.7\%) patients fulfilled ACR 2010 criteria for fibromyalgia, $5(0.67 \%)$ patients fulfilled CASPAR criteria for PsA, $3(0.4 \%)$ patients fulfilled 2015 classification criteria for Gout.
Family history of RA was present in $4(10.52 \%)$ patients and AS in $9(32.1 \%)$ patients. Co-morbidities as hypothyroid were present in $132(17.86 \%)$ patients, hypertension in $88(11.90 \%)$ and diabetes mellitus in $168(22.73 \%)$ patients. Limitation of study - This study was not community based and only those patients who had persistent symptoms were recruited. Those patients whose symptoms of CHIKV infection subsided were not included.

Conclusions: CHIKV is a worldwide epidemic which precipitates chronic inflam matory rheumatological diseases.

Acknowledgements: Nil

Disclosure of Interest: None declared

DOI: 10.1136/annrheumdis-2018-eular.6722

\section{SAT0384 EVOLVING PATTERNS OF REACTIVE ARTHRITIS}

K.M. Hayes ${ }^{1}$, R. Hayes ${ }^{2}$, J. Pope ${ }^{3} .{ }^{1}$ Schulich School of Medicine, Western University, London; ${ }^{2}$ Department of Genetics and Genome Biology, Hospital for Sick Children, Toronto; ${ }^{3}$ Rheumatology, St. Joseph's Health Care London, London, Canada

Background: Reactive arthritis ( $\operatorname{Re} A)$ seen by rheumatologists may be changing in frequency (less common) and severity (less than full triad of symptoms and less chronic $\operatorname{ReA}$ ). Epidemiologic changes in ReA could be due to less food borne illness, cleaner water, and possibly more rapid treatment of sexually transmitted diseases or for other unknown reasons.

Objectives: To understand rheumatologists' perspectives about changes in frequency, severity, and manifestations of ReA.

Methods: After obtaining ethics approval, 548 members of the Canadian Rheu matology Association (CRA) were surveyed via email with a reminder email. There were 6 groups of questions: demographic information, views from respondents regarding the prevalence of $\mathrm{ReA}$ (including acute, recurrent, and chronic), tests ordered to investigate suspected $\mathrm{ReA}$, treatments prescribed for $\operatorname{ReA}$ causes of ReA in their practices, and perspectives on changes in the incidence, severity and causes of ReA over time. Descriptive statistics were used to analyse the data. Results were by physician report and were not confirmed by chart audits Results: Sixty-six rheumatologists completed the survey (15.5\% response rate) The results of the survey indicated that $47 \%$ of rheumatologists believed that the incidence of $\mathrm{ReA}$ is declining, compared to $6 \%$ who thought it was increasing; and that the common causes may be changing $(39 \%$ agreed/strongly agreed with a mean $3.4 / 5$ on the Likert scale).

Acute, chronic, and recurrent ReA were all perceived to have similar frequencies in their practices. In terms of presentation, asymmetric oligoarthritis occurred in the majority of ReA seen by those surveyed (78\%). Full triad ReA (arthritis, con junctivitis, urethrits) was thought to occur in $21 \%$ of ReA cases, and patients with conjunctivitis were very likely to exhibit the rest of the triad. Similarly, patients with recurrent $\mathrm{ReA}$ were more likely to exhibit the full triad (43\%) compared to acute or chronic $\operatorname{ReA}(14 \%)$. Rheumatologists believed that the infectious cause of $\operatorname{ReA}$ was found in only $35 \%$ of cases. The data indicate that the most common cause of ReA was 'unknown' infectious organisms, followed by gastrointestinal (GI) infections and sexually transmitted infections (STIs).

Multiple tests were ordered to investigate ReA. The three most common investigations ordered by respondents included testing for chlamydia (66\%), C-reactive protein (CRP) (62\%), and human leukocyte antigen (HLA-B27) $(50 \%)$. Imaging was ordered by $39 \%$ of respondents with sacroiliac (SI) joint imaging ordered by $21 \%$, X-rays of the affected joints by $15 \%$, and other imaging by $7.5 \%$. Figure 1 shows these results.

Treatments used for ReA varied, as shown in figure 2 . The most common treatments for ReA were nonsteroidal anti-inflammatory drugs (NSAIDS) (97\% frequently or always used), intra-articular corticosteroid injections (65\% frequently or always used), and disease-modifying antirheumatic drugs (DMARDs) (45\% frequently or always used). Sixty-six percent said they used tumour necrosis factor alpha inhibitors (TNFi) at least occasionally in chronic ReA. 